\title{
Chitosan/Alginate/Gellan Gum Hybrid Hydrogel as a Vehicle for Controlled Release of Drug
}

\author{
Jiunn-An Pan ${ }^{1,2, ~ *, ~ H s u a n-L i a n g ~ L i u ~}{ }^{3}$, Ko-Shao Chen ${ }^{4}$ \\ ${ }^{1}$ Graduate Institution Engineering Technology-Doctoral, National Taipei University of Technology, Taipei, Taiwan \\ ${ }^{2}$ New Taipei Municipal Yonghe Junior High School, New Taipei City, Taiwan \\ ${ }^{3}$ Department of Chemical Engineering and Biotechnology, National Taipei University of Technology, Taipei, Taiwan \\ ${ }^{4}$ Department of Materials Engineering, Tatung University, Taipei, Taiwan
}

Email address:

jaint@ntpc.edu.tw (Jiunn-An Pan)

${ }^{*}$ Corresponding author

\section{To cite this article:}

Jiunn-An Pan, Hsuan-Liang Liu, Ko-Shao Chen. Chitosan/Alginate/Gellan Gum Hybrid Hydrogel as a Vehicle for Controlled Release of Drug. International Journal of Biomedical Materials Research. Vol. 7, No. 1, 2019, pp. 16-23. doi: 10.11648/j.ijbmr.20190701.13

Received: January 16, 2019; Accepted: February 20, 2019; Published: March 6, 2019

\begin{abstract}
Hybrid hydrogel was fabricated by a classic sol-gel method using EDC/NHS as crosslink reagent grafting onto the thermoplastic polyurethane (TPU), nonwoven fabric, for controlled release of drug. In this study, precursor acetic acid (AA) was used to plasma deposit on the surface of TPU to form a hydrophilic thin film. Hybrid hydrogel was investigated through scanning electron microscopy (SEM), water contact angle (WCA) measurement, Fourier transform infra-red (FTIR) spectroscopy, UV/V is spectroscopy, equilibrium swelling ratio, MTT assay and drug delivery system studies. This polyelectrolyte complexes (PECs) formed hydrogel, $\mathrm{pH}$-sensitive type, was evaluated at $\mathrm{pH}$ value of 1.2 and 7.4 of buffer solution and at temperature of $37^{\circ} \mathrm{C}$ to observe its rate of swelling and drug release features with caffeine. Moreover, the mechanism of caffeine release from membrane devices $(n=0.58)$ are anomalous transport, non-Fickian diffusion, the value of $n$ lies between 0.43 and 0.85 . It has an excellent release ratio up to about $90 \%$ absorption cumulative amounts of caffeine at $\mathrm{pH}$ 7.4 after $8 \mathrm{~h}$ and could be a beneficial carrier for fragile drugs.
\end{abstract}

Keywords: TPU, Acetic Acid Plasma, EDC/NHS Grafting, Hybrid Hydrogel

\section{Introduction}

In the 1950s, the first developed commercial thermoplastic polyurethane, TPU, was established in the U.S. by B. F. Goodrich and in the Germany by Bayer-Fabenfabriken. TPU commonly made by polyol, diisocyanate and a chain extender. It is a block copolymer composed of soft and hard blocks constituting a two-phase micro-structure. One is the soft block consists of polyester or polyether given their elastomeric and flexibility properties, another is the hard block built out of isocyanate and a chain extender offer TPU its high modulus, toughness and tensile strength. Therefore, this nonwoven has some advantages such as vast surface area [1], transparent, weathering resistance [2], heat and aging resistance [3], elasticity [4], water-proof [5].

Chitosan/ Alginate / Gellan gum hybrid hydrogel is a three-dimensional network hydrophilic heterpolymer, polysaccharide. First of all, chitosan is a linear cationic polysaccharide [6] and primary aliphatic amine: $\beta(1,4)$ linked 2-amino-2-deoxy-D-glucan and 2-acetamido-2-deoxyD-glucan $[7,8]$. It is apt to soluble in acidic aqueous solution and to form positive charges $\left(\mathrm{NH}_{3}^{+}\right)$at acidic $\mathrm{pH}(\mathrm{pH}<6.5)$ $[9,10]$. Main sources of chitosan are crabs, shrimps, molluscs, and squids $[11,12]$. Generally speaking, chitosan is usually described in detail by degree of deacetylation (DD) and average molecular weight $(\mathrm{Mw})$. Chitosam to form polyelectrolyte complexes (PECs) [13, 14], frequently used opposite charge polysaccharide [15] such as pectin, alginate [16], gellan gum [17] and $\kappa$-carrageen [18] in order to avoid chitosan drawback in the strong acid environment, and to promote water-absorbing, swelling phenomena, and lower critical solution temperature (LCST).

Alginate is a linear anionic polysaccharide, consisting of $(1,4)$ linked $\alpha$-L-guluronic acid $(\mathrm{G})$ and $\beta$-D-mannuronic acid 
(M) two units, were arranged in homopolymeric M- and Gblocks or alternating MG-blocks difference between the both proportion. Alginate is generally obtained from bacteria and seaweed [19]. It is a mono-valence, and water-soluble copolymer. Its salt transformed into water-insoluble salt due to the addition of divalent ions [20] such as calcium [21], barium, and strontium. Especially, calcium ion has not equal affinity between mannuric acid and guluronic acid, bound to the polyguluronate chain series to form "egg-box" shape. Therefore, alginate's structure has two units demonstrated that the polyguluronate $(\mathrm{GG})$ residues much tougher than polymannuronate (GG) residues and both tougher than altering $(\mathrm{MG})$ residues. Its structure is unstable and easy to drawback under strong acid environment $[22,23]$. Otherwise, gellan gum is added to hydrogel to avoid drawback, based on it is able to maintain stable in the lower acid medium. Gellan gum is a linear anionic polysaccharide, has some excellent advantages such as hydrophilic, biodegradable, biocompatible, non-toxic, and cheap. It produced by the microorganism Sphingomonas elodea [24] composed of repeating tetrasaccharide (1,3- $\beta$-D-glucose, $1,4-\beta$-Dglucuronic acid, 1,4- $\beta$-D-glucose, 1,4- $\alpha$-L-rhamnose) units, in the molar ratios 1:2:1 ( $\alpha$-L-rhamnose, $\beta$-D-glucose, and $\beta$ D-glucuronate) and possessed one carboxyl side group [25]. Native gellan gum has lower LCST (lower critical solution temperature), soft, and easy to reform gels [26, 27]. Contrary, the deacetylated one is rigid and brittle gel [28]. Gellan gum is efficiently able to form gel by the addition of cation $\left(\mathrm{Na}^{+}\right.$, $\left.\mathrm{k}^{+}, \mathrm{Ca}^{2+}, \mathrm{Cu}^{2+}\right)$ and characteristics of changeful texture depend on the gelation conditions [29, 30]. This hydrogel has usually used in the food additive agent (used as a thicker, stabilizer, and emulsifier), cosmetic, and pharmaceutical used. Hence, this polyelectrolyte complexes has usually been used to control drug release [31-34]. In this study, caffeine was used as a release drug owing to its cheap, harmless, convenient and productive medicine for pharmacotherapy [35-38].

Lately, nonwoven fabric of TPU has been extensively used in various fields (bioreactor, biomedical material, biosensor), which has many advantages including mechanical strength, large surface area, resistant acid and base and bioavailability. In this study, it is used as a medicine carrier substrate, hydrophobic fabric. Hence, TPU surface is hard to bind with polar surface of material (collagen, enzyme, cell, chitosan), leading easy to peel off objective. For this reason, plasma technologies [39] (corona [40], glow [41], photo-grafting, UV induced and graft polymerization $[42,43]$ ) have been used to modify the surface of TPU. It is a very effective way to promote hydrophilicity of TPU surface by applying free radicals, peroxides (OOR, $\mathrm{OOH})$, and functional groups $\left(\mathrm{SiO}_{2}, \mathrm{CH}_{3}, \mathrm{OH}, \mathrm{COOH}, \mathrm{C}=\mathrm{O}\right.$, and $\mathrm{NH}_{2}$ [44]) introduced by plasma or grafting of gas or monomers [45] such as $\mathrm{O}_{2}$ [46, 47], $\mathrm{He}, \mathrm{Ar}[48,49], \mathrm{N}_{2}, \mathrm{CH}_{4}, \mathrm{C}_{2} \mathrm{H}_{2}, \mathrm{CO}_{2}$, acetic acid, acrylic acid [50, 51], N-isopropylacrylamide (NIPAAm) [52], hexamethyldisilazane (HMDSZ), isopropanol. This hybrid hydrogel made from Chitosan, alginate, and gellan gum three element hydrogel and formed a three-dimensional network hydrophilic polymer. TPU membrane surface was modified by PECVD [53] or lysine [54, 55]. Coming after, Nhydroxysuccinimide (NHS) and 1-ethyl-3-(3-dimethyl aminopropyl) carbodiimide hydrochloride as coupling agents [56- 59] are used to crosslink hydrogel (carboxyl side group) and TPU membrane, and then Caffeine loaded hydrogelbased polymeric film for controlled drug delivery. Finally, the surface characterizations of film and drug delivery system were made use of water contact angle measurement, scanning electron microscopy, Fourier transform infra-red spectroscopy, UV/Vis spectroscopy, swelling behavior of films and MTT assay were also tested

\section{Materials and Methods}

\subsection{Material and Reagents}

Deacetylated gellan gum, sodium alginate (MW=10,000 600,000), acetic acid, 1-ethyl-3-(3-dimethyl aminopropyl) carbodiimide. $\mathrm{HCl}$ (EDC), polyvinylpyrrolidone (PVP) were provided by Wako Pure Chemical Company, USA. Chitosan (deacetylation degree $>$ 93\%, M.W. $=190 \sim 310 \mathrm{~K}$ ) was purchased from Aldrich Company. Caffeine anhydrous and sodium tripolyphosphate (Na-TPP) were obtained from Sigma Company. Thermoplastic polyurethane fabric nonwoven (TPU) was supplied by Taiwan Textile Research Institute. Argon (purity $>99 \%$ ), oxygen (purity $>99 \%$ ) and carbon dioxide were purchased from Sanfu chemical Company. Nhydroxysuccinimide (NHS, purity $>97.0 \%$ ) was obtained from Fluka. Trypan blue stain, Minimum essential medium Eagle, Dimethyl sulfoxide, Sodium bicarbonate and 3-(4, 5Dimethylthiazo-2-yl)-2, 5-diphenyl tetrazolium bromide were purchased by Sigma. CCD-966SK cell line (Human skin fibroblast) was obtained from Bioresource Collection and Research Center, Hsinchu, Taiwan. MEM non-essential amino acids solution, Penicillin, Streptomycin, L-glutamine, and Dulbecco's Modified eagle medium were purchased from GIBCO/BRL, USA. Fetal bovine serum, and TrypsinEDTA were obtained from HyClone, USA. All of the other chemicals and reagents were of analytical grade and used without further purifications.

\subsection{Cold Plasma of Surface Treatment}

Samples (TPU, thickness: $0.2 \mathrm{~cm}$ ) were cut into the pieces $(1 \mathrm{~cm} \times 1 \mathrm{~cm})$ as substrates, ultrasonically cleaned in alcohol and deionized water to remove film surface impurities and dried at room temperature. Advanced plasma system (Model PD-2S plasma deposition system manufactured by SAMCO Company) with $13.6 \mathrm{MHz}$ radio frequency generator and a bell jar type reactor were used to activate the surfaces of the films. All process of the cold plasma treatment was described in detail in previous study [42]. Foremost, samples were treated by placed over the electrode, a horizontal holder, and the chamber was pumped to a base pressure of 30 mtorr before the cold plasma treatment which was stabilized. Next, the acetic acid (AA) monomers were introduced into the 
chamber (pressure: 50mtorr, and at a discharge power of $40 \mathrm{~W}$ ) for treatment times of $1,5,10,15,30$ minutes, respectively, for sample surfaces to produce $\cdot \mathrm{COOH}$ group.

\subsection{Hybrid Hydrogel Coupling to Surface of Treated TPU by $\mathrm{EDC} / \mathrm{NHS}$}

The AA-treated film was immersed in $2 \mathrm{mM}$ EDC/NHS aqueous solution (0.1M MES buffer, $\mathrm{pH}$ 5-6) at room temperature for $2 \mathrm{~h}$, and then washed the $\mathrm{pH}$ 7.2-7.5 with $0.05 \mathrm{M}$ phosphate buffer immediately before reaction to the hybrid hydrogel. Allowing EDC/NHS-treated films reacted with the hybrid hydrogel for $2 \mathrm{~h}$ at room temperature. After grafting treatment, and washed with deionized water to remove un-reacted reagent and dried them at room temperature for overnight. For the preparation of the alginate/gellan gum/chitosan hybrid hydrogel, chitosan power was dissolved at $1 \%(\mathrm{w} / \mathrm{v})$ concentration in aqueous acetic acid $(1 \% \mathrm{v} / \mathrm{v})$ with constant stirring at a room temperature. Polyvinylpyrrolidone (PVP) was dissolved in deionized water $(0.5 \%)$. Both chitosan and PVP solutions were mixed and stirred at room temperature for $2 \mathrm{~h}$. Alginate was dissolved at $1 \%(\mathrm{w} / \mathrm{v})$ concentration in the deionized water with constant stirring at a room temperature. Gellan gum was dissolved at $1 \%(\mathrm{w} / \mathrm{v})$ concentration in the deionized water with constant stirring at a temperature of $80^{\circ} \mathrm{C}$ for $2 \mathrm{~h}$. The AA-treated films were soaked in an aqueous solution of mixed chitosan/PVP, alginate and gellan gum solution in the ratio $(\mathrm{w} / \mathrm{v}) 1: 1: 0,1: 1: 1,1: 1: 2,0: 1: 0$, and 1: $0: 4$ at a temperature of $50^{\circ} \mathrm{C}$ for $2 \mathrm{~h}$, and put them into $1 \%$ Na-TPP aqueous solution for overnight. Next, the grafted films were washed with distilled water overnight to remove the unbinding reactant.

\subsection{Surface Characterization}

The surface morphology of the films was observed using scanning electron microscope (SEM, JSM 5600). The water contact angle (WCA) of original and modified films were measured by CCD camera (Goni-meter type G-1 made by
ERMA Optical Works Company). The Fourier transform infrared spectrometer (Jasco FT/IR-6200) was used to analyze the surface functional groups after the plasma modification, graft polymerization, and immobilization of hybrid hydrogel (chitosan, alginate, and gellan gum). The grafting density was also measured.

\subsection{Swelling Characteristics}

The swelling behaviors of films were examined in simulated gastric fluid $(\mathrm{pH} 1.2,0.1 \mathrm{M} \mathrm{HCl} / \mathrm{NaCl}$ buffer solution, SGF), and simulated colonic fluid ( $\mathrm{pH} 7.4,0.05 \mathrm{M}$ phosphate-buffered SCF). Films of known weight were successively immersed in $10 \mathrm{ml}$ of SGF, and SCF at a temperature of $37^{\circ} \mathrm{C}$. The swollen films were removed to a fresh $10 \mathrm{ml}$ buffer solution and weight at fitting interval, sucking them with filter paper to remove the surface film of liquid adherence at once. The swelling ratio (SR) of film was calculated, using the following equation:

$$
\mathrm{SR}(\%)=\left[\left(\mathrm{W}_{1}-\mathrm{W}_{0}\right) / \mathrm{W}_{0}\right] \times 100 \%
$$

Where $W_{1}$ is the weight of the swollen test film at fitting intervals and $\mathrm{W}_{0}$ is the dried weight of film.

\subsection{Drug Release Test}

The Caffeine profile of film was measured for the purpose of impersonation gastrointestinal tube (GIT, included: $\mathrm{pH}$ 1.2, in SGF, and $\mathrm{pH} 7.4$, in SCF) from stomach to colon. The caffeine-loaded film was equilibrated in a solution of $30 \mathrm{mg}$ drug and $1 \mathrm{~L}$ deionized water (30ppm) [60]and incubated at $37^{\circ} \mathrm{C}$ for $24 \mathrm{~h}$. The drug release test was carried through by transferring previously incubated drug hydrogel into a $10 \mathrm{ml}$ buffer solution at $37^{\circ} \mathrm{C}$ and at fitting interval. The film was constantly removed and transferred into a fresh $10 \mathrm{ml}$ buffer solution at different time interval. Capacity of the caffeine in film was determined at $273 \mathrm{~nm}$ by making use of a UV-Vis spectrophotometer (JASCO 1700).

$$
\text { Loading efficiency }(\%)=\frac{\text { Actual amount caffeine in hydrogel }}{\text { Theoretical yield of caffeine loaded hydrogel }}
$$

\subsection{Cell Culture Protocol}

CCD966SK cell line (BCRC 60153) was obtained from Bioresource Collection and Research Center (Hsinchu, Taiwan). Cells were seeded at a density $3.0 \times 10^{4}$ cells per well into 24well plates and grown in Dulbecco's Modified Eagle's Medium supplemented with $10 \%$ fetal bovine serum, $100 \mathrm{Mm}$ non-essential amino acid, $100 \mathrm{U} / \mathrm{ml}$ penicillin and $100 \mathrm{U} / \mathrm{ml}$ streptomycin mixed antibiotics, $\mathrm{NaHCO}_{3}$ and $2 \mathrm{mM} \mathrm{L-}$ glutamine. All cell cultures were maintained in a humidified atmosphere of $5 \% \mathrm{CO}_{2}$ at $37^{\circ} \mathrm{C}$ and exposed to hydrogel films for 1 to 3 days. Assessment of cell viability was determined by MTT assay based on live cell's mitochondrial (succinate dehydrogenase) reduction of yellow MTT tetrazolium salt to blue purple formazan crystal. The medium was regularly changed and incubated with MTT reagent
$(5 \mathrm{mg} / \mathrm{ml})$ at $37^{\circ} \mathrm{C}$ for $4 \mathrm{~h}$. The reaction was stopped by adding dimethyl sulfoxide (DMSO) and measured optical density (Multiskan Spectrum Microplate Spectrophotometer) at 540nm $\left(\mathrm{OD}_{540}\right)$.

\section{Results and Discussions}

\subsection{Surface Morphology Survey}

Figure 1 SEM images of the surface morphologies of (a) un-modified and (b) hybrid hydrogl-treated TPU. The surface of un-modified TPU film revealed a lot of rough squamae. After acetic acid plasma treatment and grafting alginate/chitosan/gellan gum hybrid hydrogel, the treated surface of TPU was apparently changed as Figure 1(b) shown. Both of SEM images created diversely a 
transformative surface of TPU from coarse to flat structure. Therefore, surface of TPU film could be modified by plasma treatment and gelatin, has some alterations.
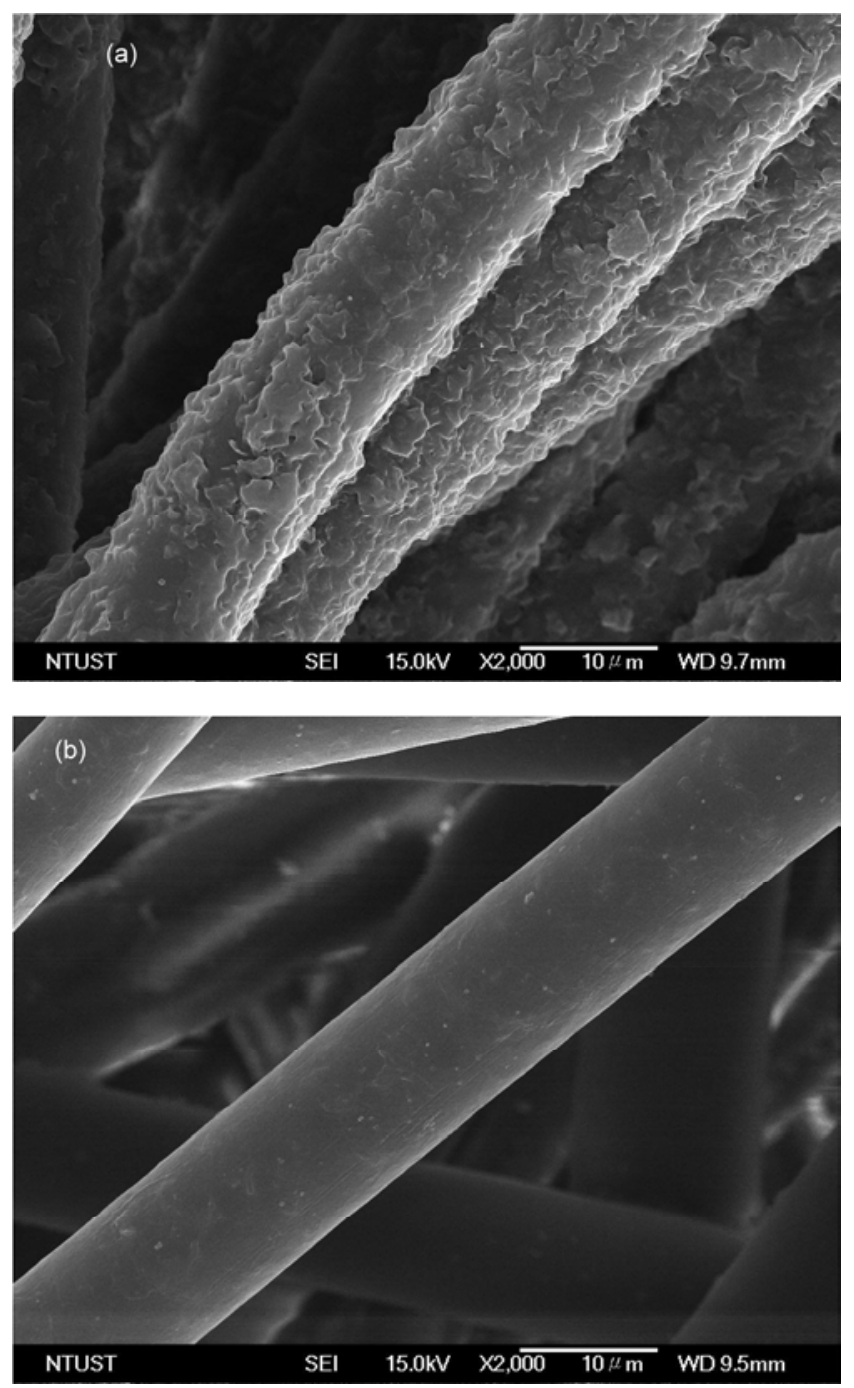

Figure 1. SEM of (a) un-modified and (b) hybrid hydrogl-treated TPU film, hybrid hydrogl in the ratio, Chitosan: Alignate:Gellan gum $=1: 1: 2(w / v \%)$

\subsection{Wettability of Modified Surface}

TPU thin film, nonwoven fabric, has specifically a hydrophobic property. Figure 2 (b) shown water contact angle of TPU surface has been obviously changed owing to plasma treated. Water contact angle (WCA) of TPU film was measured by the sessile drop method with distilled water at room temperature. The data were recorded by a CCD camera (Goni-meter type G-1, ERMA Optical Works), which of unmodified TPU is $116.70^{\circ}$. After plasma deposition of acetic acid, at power of $40 \mathrm{~W}$ and 50 mtorr for 30 minutes, WAC is $106.4^{\circ}$, demonstrated hydrophilic leaning was due to cover with acetic acid. Of TPU has dramatically changed by acetic acid plasma treatment to form hydrophilic film.
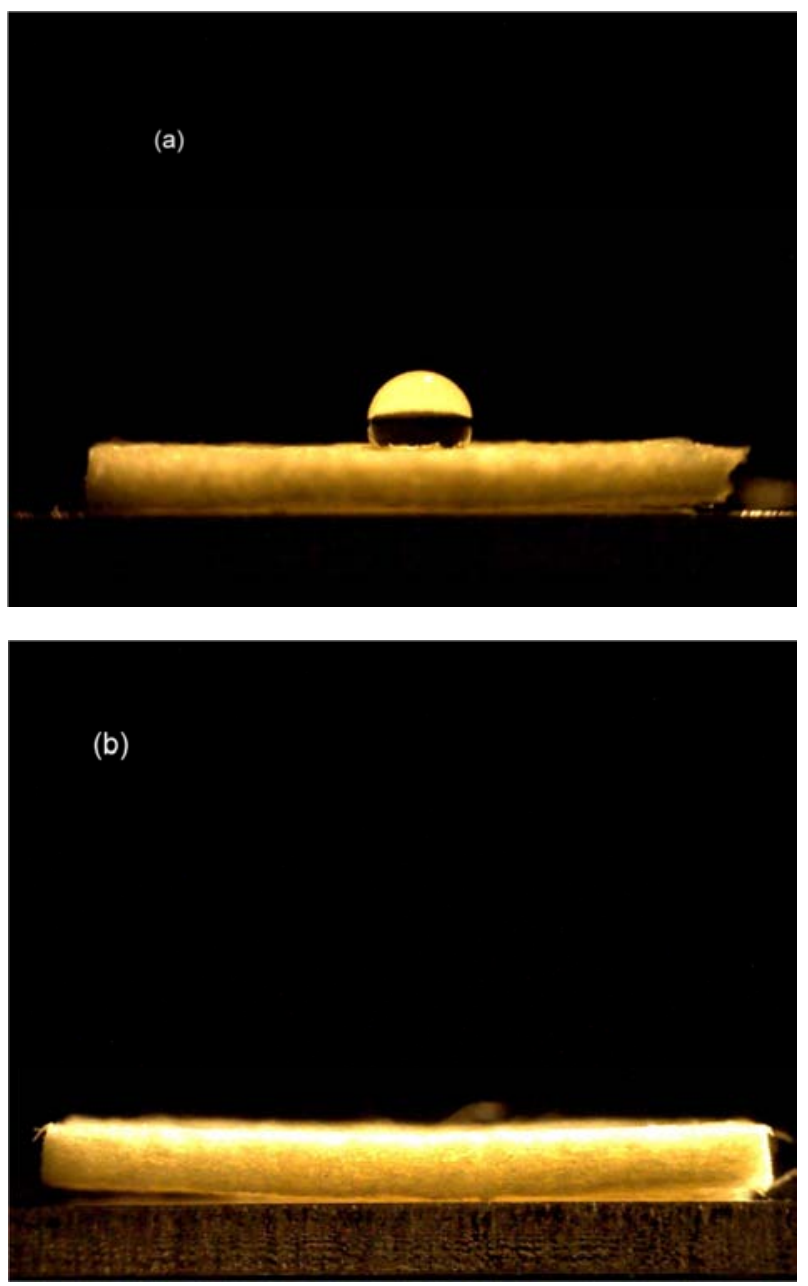

Figure 2. Water Contact Angle of TPU due to acetic acid Plasma treatment (a) un-modified $\left(116.7^{\circ}\right)$ (b) AA-treated acetic acid TPU $\left(0^{\circ}\right)$. plasma treatment condition: $40 \mathrm{~W}, 50$ mtorr acetic acid, and treatment time $=$ 30minutes.

\subsection{FTIR Spectral Analysis}

Figure 3 illustrated the FTIR spectra of TPU of acetic acid plsma and hybrid hydrogel treatment were composed of chitosan, alginate and gellan gum. Alginate and gellan gum represented one carboxyl $(\mathrm{C}=\mathrm{O})$ peak at $1640 \mathrm{~cm}^{-1}$ slightly shafted to $1653.1 \mathrm{~cm}^{-1}$, the $-\mathrm{C}-\mathrm{O}-\mathrm{C}$ - peak at $1221.21 \mathrm{~cm}^{-}$ ${ }^{1}$ strentching due to carboxylic acid. TPU has characteristic sharp peak at $1157.5 \mathrm{~cm}^{-1}, 1538.4 \mathrm{~cm}^{-1}, 1722.8 \mathrm{~cm}^{-1}$ owing to $-\mathrm{C}-\mathrm{N}$ stretching vibration and $-\mathrm{N}-\mathrm{H}$ bending vibration overlapped, and -HNCOO- stretching vibration, respectively. The peak at $2848.8 \mathrm{~cm}^{-1}$ and $2910.8 \mathrm{~cm}^{-1}$ exhibited asymmetric and symmetric stretching vibration of $-\mathrm{CH}_{2}$. The characteristic peak of $-\mathrm{CO}$ stretching vibration of acetic acid was observed at $1061.6 \mathrm{~cm}^{-1}$ in Figure 3 and at 932.2, 1451.9 $\mathrm{cm}^{-1}$ assigned to $-\mathrm{C}-\mathrm{H}$ bending and scissoring vibration. Hence, FTIR spectra of hybrid hydrogels for chitosan demonstrated in Figure 3, shown sharp peak in at $3210.4 \mathrm{~cm}^{-}$ ${ }^{1}, 1653.1,1577 \mathrm{~cm}^{-1}$ are due to the $\mathrm{O}-\mathrm{H}$ bending vibration overlapped with the $\mathrm{N}-\mathrm{H}$ stretch vibration, the amide $\mathrm{I}(\mathrm{C}=\mathrm{O}$ stretching vibration of acetyl group), amide II (N-H shift stretching vibration), respectively. 


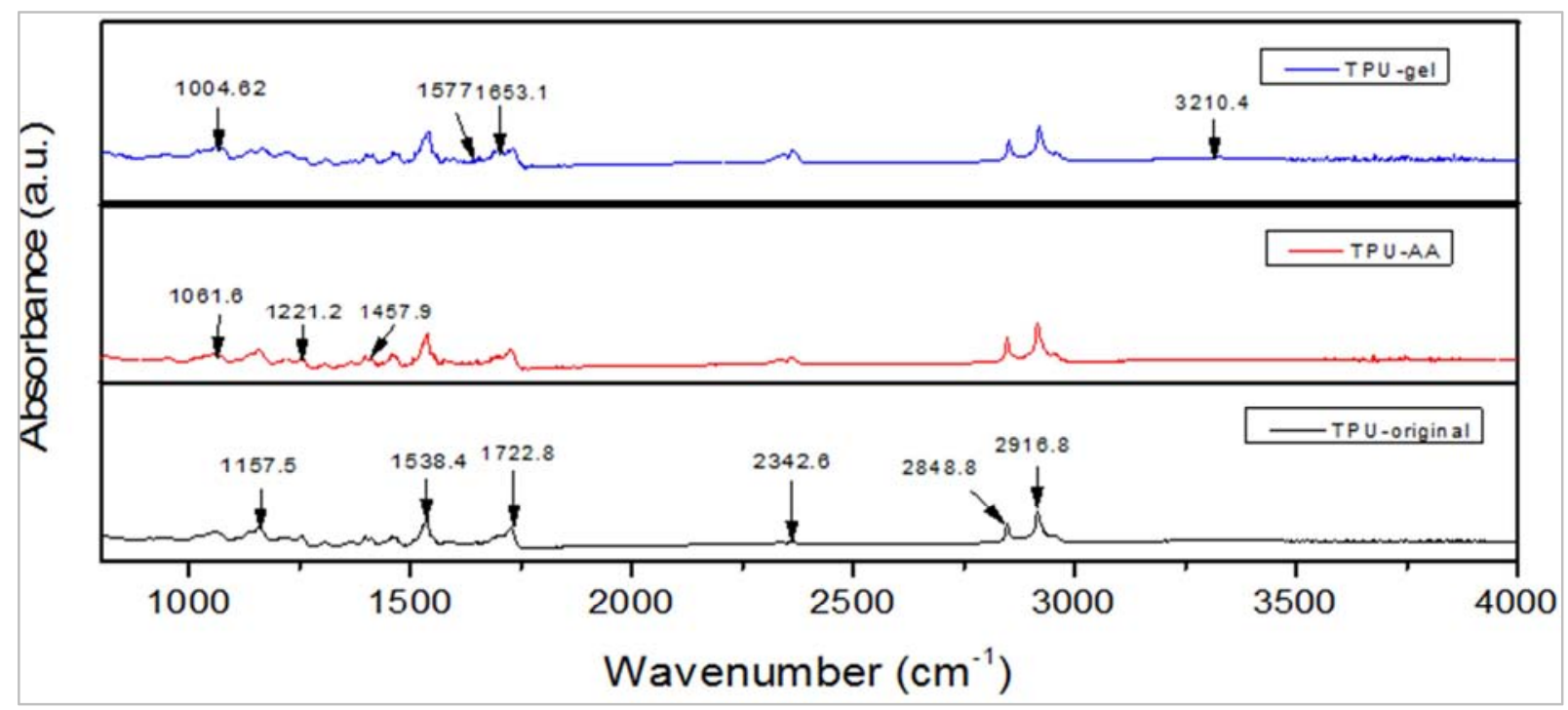

Figure 3. FTIR spectra of TPU of acetic acid plasma and hybrid hydrogel treatment, rato of Chitosan: Alginate: Gellan gum is 1: 1:2 (w/v \%).

\subsection{Equilibrium Swelling Study}

Effect of hydrogel variation compositions (w/v \%) of TPU film are due to equilibrium swelling in alkaline and acid conditions shown in Table 1. Swelling behavior of hybrid hydrogel was measured with water uptake at a fitting interval. Next, the dry copolymer hydrogel was placed in buffer solutions with $\mathrm{pH} 1.2$ and $\mathrm{pH} 7.4$ at $37^{\circ} \mathrm{C}$ for $8 \mathrm{~h}$ to be allowed hybrid hydrogel to achieve equilibrium swelling state. The weight of hydrogel film is $0.167 \pm 4.71 \times 10^{-05}$ $\mathrm{mg} / \mathrm{cm}^{2}$ (mean $\pm \mathrm{SD}, \mathrm{N}=3$ ). Table 1 showed a variety of SR (\%) for hybrid hydrogel, investigated that contained alginate $(-\mathrm{COOH})$, chitosan $\left(-\mathrm{NH}_{2}\right)$ and gellan gum $(-\mathrm{COOH})$ of different ratio at $37^{\circ} \mathrm{C}$. In acidic environment, $\mathrm{pH} 1.2$, swelling behavior of the hydrogel film was lower, the performance exceedingly increased in alkaline environment,
$\mathrm{pH7}$ 4. Under the lower $\mathrm{PH}$ environment, $\mathrm{pH} 1.2$, the $-\mathrm{NH}_{2}$ group kept to proton, owing to $\mathrm{H}^{+}$ion promoted and raised electrostatic repulsive force. Consequently, hybrid hydrogel $\left(-\mathrm{NH}_{3}{ }^{+},-\mathrm{COOH}\right)$ could be to swell up, $-\mathrm{COOH}$ part of hydrogel was obviously inhibited. At higher $\mathrm{pH}$ value of alkaline environment, $-\mathrm{COOH}$ part of hydrogel ionized to $\mathrm{COO}{ }^{-}$that exerted electrostatic repulsive force, and $-\mathrm{NH}_{2}$ part of hydrogel was inhibited. Accordindly, it resulted in volume of alteration at different $\mathrm{PH}$, this swelling behavior of the hydrogel network depended on ratio of alginate, chitsan and gellan gum. The pH-sensitive experiment of hybrid hydroge of swelling ratios found them up to maximum after $8 \mathrm{~h}$. When hybrid hydrogel, alginate: chitosan: gellan gum $=$ 1: 1: $2(\mathrm{w} / \mathrm{v} \%)$, operated at $37{ }^{\circ} \mathrm{C}$ for about $8 \mathrm{~h}$, the SR \% ratio was able to gain a maximum divergence.

Table 1. Effect of hydrogel variation compositions ( $w / v \%)$ of TPU film is due to the equilibrium swelling in alkaline and acid conditions. (mean $\pm S D, N=3)$.

\begin{tabular}{|c|c|c|c|c|c|c|}
\hline \multicolumn{5}{|c|}{ Hydrogel Types } & \multicolumn{2}{|c|}{ Equilibrium swelling (\%) } \\
\hline Chitosan & : & Alginate & $:$ & Gellan gum & pH1.2 & pH7.4 \\
\hline 1 & : & 1 & $:$ & 0 & $185.47 \pm 25.19$ & $332.93 \pm 35.60$ \\
\hline 1 & : & 1 & : & 1 & $166.40 \pm 10.74$ & $276.80 \pm 16.25$ \\
\hline 1 & : & 1 & : & 2 & $166.00 \pm 14.06$ & $355.45 \pm 20.11$ \\
\hline 1 & : & 0 & $:$ & 0 & $139.20 \pm 23.83$ & $320.13 \pm 19.82$ \\
\hline 1 & : & 0 & : & 4 & $200.93 \pm 74.95$ & $276.67 \pm 30.14$ \\
\hline 0 & : & 0 & $:$ & $0 *$ & 0 & 0 \\
\hline
\end{tabular}

*un-reated TPU

\subsection{Caffeine Release and Cytotoxicity Studies}

Figure 4 showed that the caffeine release curves of various $\mathrm{pH}$ investigated at $37^{\circ} \mathrm{C}$. Cumulative release was higher amount of caffeine due to suitable ratio of hybrid hydrogel, Chitosan: Alginate: Gellan gum $=1: 1: 2(\mathrm{w} / \mathrm{v} \%)$. These films loaded drug amount are $6.24 \times 10^{-4} \pm 2.24 \times 10^{-5} \mathrm{mg} / \mathrm{cm}^{2}$ (mean $\pm \mathrm{SD}, \mathrm{N}=3$ ). Loading efficiency $(\%)$ is $92.45 \pm 1.05 \%$ (mean $\pm \mathrm{SD}, \mathrm{N}=3$ ). Table 2 exhibited drug release from caffeine-loaded hybrid hydrogel followed non-Fickian model transport mechanism in view of diffusion exponent at PH1.2 and $\mathrm{PH} 7.4$ were 0.57 and 0.58 , respectively. The drug released behavior was due to volume alteration to form stress effect rather than non-Fickian diffusion mechanism. Cytotoxicity assay of hybrid hydrogel was done by MTT testing. Figure 5 demonstrated that plot of viability of \% CCD966SK cells line for 24 72h. The hybrid hydrogel is a polyelectrolyte complexes (PECs) formed hydrogel. It has some positive charges and characteristics of smooth surface texture could be apt to adhered cell. Therefore, the cell viability values were found out to be $126.39 \pm 0.04 \%$ and 
$192.36 \pm 0.17 \%$ (mean $\pm \mathrm{SD}, \mathrm{N}=3)$ for hybrid hydrogel compared with control $\left(3 \times 10^{4} \mathrm{cell} / \mathrm{well}\right)$.

Table 2. Release kinetic data of Caffeine loaded hydrogel (w/v \%) of TPU film.

\begin{tabular}{llll}
\hline Hydrogel Types & \multicolumn{2}{l}{ Korsmeyer-Peppas Model } & Caffeine cumulative release rate (\%) \\
\cline { 2 - 4 } Chitosan:Alginate:Gellan gum 1:1:2 & $\mathbf{k}$ & $\mathbf{N}$ & (mean \pm SD, $\mathbf{N}=\mathbf{3}$ ) \\
\hline pH 1.2 & 0.069 & 0.57 & $42.30 \pm 2.54$ \\
pH 7.4 & 0.173 & 0.58 & $88.77 \pm 2.65$ \\
\hline
\end{tabular}

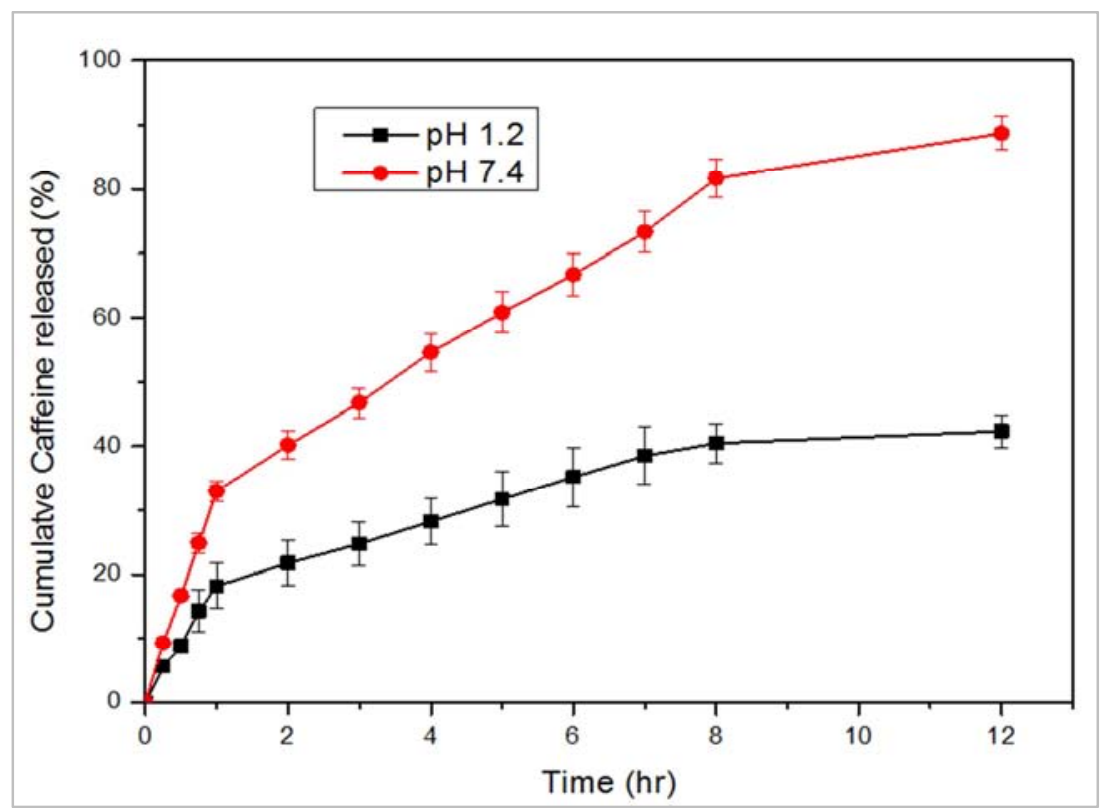

Figure 4. The caffeine release curves of various pH investigated at temperature of $37^{\circ} \mathrm{C}$, ratio of Chitosan: Alginate: Gellan gum is 1: $1: 2$ (w/v \%).

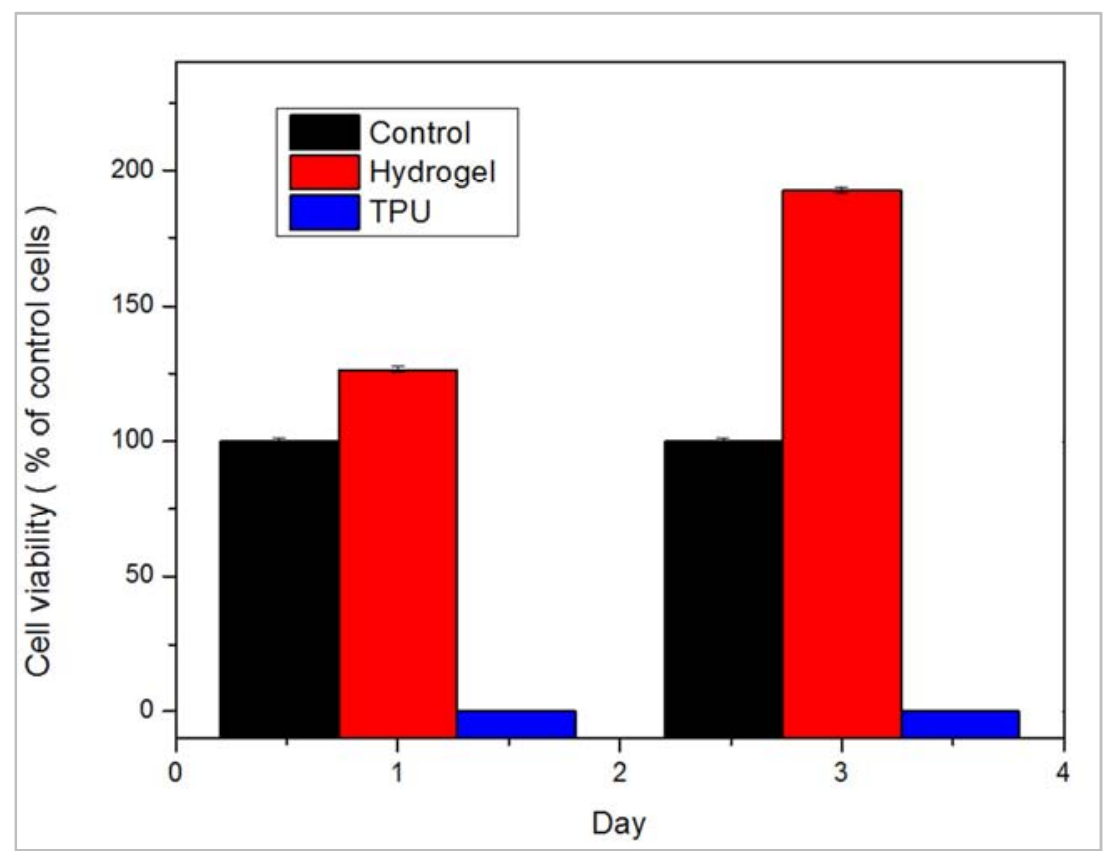

Figure 5. In vitro cytotoxicity analysis of the CCD-966SK cells on the hydrogel, Chitosan: Alginate: Gellan gum =1: 1: $2(w / v \%)$. CCD966SK cells $\left(3 \times 10^{4}\right.$ cells/well) were incubated on the hydrogel for 1 and 3 days, respectively. (mean $\pm S D, N=3$ ).

\section{Conclusions}

Caffeine is widely used to consume as psychoactive drug. In this study, pH-sensitive hybrid hydrogel of chitosan, alginate and gellan gum were prepared by ionotropic gelatification to load caffeine, which concentration ratio of hybrid hydrogel has a crucial influence on the swelling rate and release performances. Surface of TPU nonwoven film was hydrophobic which binding polar material is difficult, so utilized acetic acid plasma treatment improved hydrophilic of 
surface. Also, hybrid hydrogel was settled on the surface of TPU nonwoven film by EDC/NHS crosslinking reagent. This $\mathrm{pH}$-sensitive hybrid hydrogel is a three-dimensional network and hydrophilic polyelectrolyte, polysaccharide, could tolerate lower $\mathrm{pH}$ to release caffeine which avoided being destroyed. It is based on gellan gum could not be split up in strong acid environment. Therefore, drug could be almost performed to target, released cumulative ratio up to about $90 \%$ absorption amounts of caffeine after $8 \mathrm{~h}$. This drug release system followed non-Fickian type transport mechanism and electrostatic repulsive force. This results exposed that was able to attenuate the release of caffeine at the acid medium, $\mathrm{pH} 1.2$, to be suitable for drug release at the alkaline environment, $\mathrm{pH} 7.4$, minimized the loss of caffeine at the acid medium to the first-pass metabolism and improved on bioavailability.

\section{Acknowledgements}

This project was funded by the National Science Council, Taiwan (M. O. S. T, No. 106-2923-E-036-001).

We thank Mr. J. F. Lin for providing technical support and all authors declare no conflicts of interest.

\section{References}

[1] Qi-Wei Lu and Christopher W. Macosko. Promoting Adhesion to Thermoplastic Polyurethane (TPU) by Amine Functional Polypropylenes. Polymeric Materials: Science \& Engineering 2003; 89:844-7.

[2] Emre Basturk, Serfullas Madakbas, Memet Vezir Kahraman. Improved Thermal Stability and Wettability Behavior of Thermoplastic Polyurethane/Barium Metaborate Composites. Materials Research 2016; 19:434-9.

[3] A. Boubakri, N. Guermazi, K. Elleuch, et al. Study UV-aging of thermoplastic polyurethane material. Materials Science and Engineering A 2010; 527:1649-54.

[4] Y. Jia, Z. Jiang, X. L. Gong, et al. Creep of thermoplastic polyurethane reinforced with ozone functionalized Carbon nanotubes. eXPRESS Polymer Letters $2012 ; 6: 750-8$.

[5] Katsuji Matsunaga, Kyoko Sato, Masahiro Tajima, et al. Gas Permeability of Thermoplastic Polyurethane Elastomers. Polymer Journal 2005; 37:413-7.

[6] E. L. Larionova, T. N. Kalinina, T. I. Chufarovskaya, et al. Fibre, Film, and Porous Materials Based on Chitosan. Fibre Chemistry 1995; 27:392-6.

[7] Sunil A. Aginhotri, Nadagouda N. Mallikarjana, Tejraj M. Aminabhavi. Recent advances on chitosan-based micro-and nanoparticles in drug delivery. Journal of Controlled Release 2004; 100:5-28.

[8] S. Demarger-Andre \& A. Domard. Chitosan carboxylic acid salts in solution and in solid state. Carbohydrate Polymer 1994; $23: 211-9$.

[9] Sang Yond Nam, Young Moo Lee. Pervaporation and properties of chitosan-poly (acrylic acid) complex membranes. Journal of membrane science 1997; 135:161-71.
[10] M. V. Shamov, S. Yu. Bratskyaya, and V. A. Avoramenko. Interaction of Carboxylic acid with Chitosan: Effect of $\mathrm{pK}$ and Hydrocarbon Chain Length. Journal of Colloid and Interface Science 2002; 249:316-21.

[11] Majeti N. V., Ravi Kumar. A review of chitin and chitosan applications. Reactive \& Functional Polymers 2000; 46:1-27.

[12] Narimane Mati-Baynast, Pierre-Henri Elchingeer, Helenede Baynast, et al. Chitosan as an adhesive. European Polymer Journal 2014; 60:198-212.

[13] J. Berger, M. Reist, J. M. Mayer, et al. Structure and interaction in chitosan hydrogels formed by complexation or aggregation for biomedical applications. European Journal of Pharmaceutics and Biopharmaceutics 2004; 57:35-52.

[14] George Pasparakis, Nikolaos Bouropoulos. Swelling studies and in vitro release of verapamil from calcium alginate and calcium alginate-chitosan beads. International Journal of Pharmaceutics 2006; 323:34-42.

[15] Cornelus G. de Kruif, Fanny Weinbreck, Renko de Vries. Complex coacervation of proteins and anionic polysaccharides. Current Opinion in Colloid \& Interface Science 2004; 9:340-9.

[16] Meera George, T. Emilia Abraham, Polyionic hydrocolloids for the intestinal delivery of protein drug: Alginate and chitosan - a review. Journal of controlled release 2006; 114:114.

[17] Carolina Siqueira Franco Picone, Rosiane Lopes Cunha. Chitosan-gellan electrostatic complexes: Influence of preparation conditions and surfactant presence. Carbohydrate Polymers 2013; 94:695-703.

[18] Felix N. A. Aryee, Michael T. Nickerson. Effect of pH, biopolymer mixing ratio and salts on the formation and stability of electrostatic complexes formed within mixtures of lentil protein isolate and anionic polysaccharides ( $\kappa$-carrageen and gellan gum). International Journal of Food Science and Technology 2014; 49:65-71.

[19] Wayne R. Gombotz, Siow Fong Wee. Protein release from alginate matrices. Advanced Drug Delivery Reviews 2012; 64:194-205.

[20] B. Thu, P. Bruheim, T. Espevik, et al. Alginate polycation microcapsules I . Interaction between alginate and polycation, Biomaterials 1996; 17:1031-40.

[21] Hucheng Zhang, Honghe Zheng, Qingzhi Zhang, et al. The Interaction of Sodium Alginate with Univalent Cation, Biopolymers 1998; 46:395-402.

[22] Kuen Yong Lee, David J. Moonney. Alginate: Properties and biomedical applications. Progress in Polymer Science 2012; 37:106-26.

[23] Qiang Qian, Walter Bonani, Devid Maniglio, Jie Chen, Claudio Migliaresi, Modulating the release of drugs from alginate matrices with the addition of gelatin microbeads, Journal of Bioactive and Compatible, Polymers, 2014; 29:193207.

[24] Per-Erik Jansson, Bengt Lindberg. Structural Studies of Gellan Gum, An Extracellular Polysaccharide Elaborated By Pseudomonas elodea. Carbohydrate Research 1983; 124:1359. 
[25] Cameron John Ferris and Marcinhet Panhuis. Conducting biomaterials based on gellan gum hydrogels. Soft Matter 2009; 5:3430-37.

[26] F. Yamamoto, R. L. Cunha, Acid gelation of gellan:Effect of final $\mathrm{pH}$ and heat treatment conditions, Carbohydrate Polymers 2007; 68:517-27.

[27] Edwin R. Morris, Katsuyoshi Nishinari, Marguerite Rinaudo, Gelation of gellan - A review 2012; 28:373-411

[28] Stefan kasapis, Persephoni Giannouli, Miles W. N. Hember, et al. Structure aspects and phase behavior in deacylated and high acyl gellan systems, Carbohydrate Polymers 1999; $38: 145-54$.

[29] Emako Miyoshi, Tomohisa Takaya \& Katsuyoshi Nishinari. Rheological and thermal studies of gel-sol transition ingellan gum aqueous solutions, Carbohydrate Polymers 1996; 30: 109-19.

[30] Yangchao Luo, Qin Wang. Recent development of chitosanbased polyelectrolyte complexes with natural polysaccharides for drug delivery, International Journal of Biological Macromolecules 2014; 64:353-67.

[31] Brahma N. Singh, Kwon H. Kim. Effects of divalent cations on drug encapsulation efficiency of deacylated gellan gum, Journal of Microencapsulation 2005; 22:761-71.

[32] Giorgia D'Arrigo, Gemma Navarro, Chiara Di Meo, et al. Gellan gum nanohydrogel containing antiflammatory and anticancer drugs: a multi-drug delivery system for a combination therapy in cancer treatment. European Journal of Pharmaceutics and Biopharmaceutics 2014; 87:208-16.

[33] Mrunalini Narkar, Praveen Sher, and Atmaram Pawar, Stomach-Specific Controlled Release Gellan Beads of AcidSoluble Drug Prepared by Ionotropic Gelation Method, AAPS PharmSciTech 2010; 11:267-77.

[34] Mohammed Reza Amiryousefi, Mohebbat Mohebbi, Shiva Golmohammadzadeh et al. Encapsulation of caffeine in hydrogel colloidosome: optimization of fabrication, characterization and release kinetics evaluation. Flavour and Fragrance Journal 2016; 31:163-72.

[35] Aleksandra Szopa, Ewa Poleszak, Elzbieta Wyska, et al. Caffeine enhances the antipressant-like activity of common antidepressant drugs in the forced swim test in mice, MaunynSchmiedeberg's Arch Pharmacol., 2016; 389:211-21.

[36] Puncan Turnbull, Joseph V. Rodricks, Gregory F. Mariano. Neurobehavioral hazard identification and characterization for caffeine. Regulatory Toxicology and Pharmacology 2016; 74:81-92.

[37] James G. Phillips, Jonathan Currie, and Rowan P. Ogeil. Consumption and foraging behavior for common stimulants (nicotine, caffeine), Journal of Addictive Diseases 2016; 35: 15-21.

[38] Giuseppe Grosso, Agnieszka Pajak and Fabio Galvano, Coffee, tea, caffeine and risk of depression: A systematic review and observational studies. Mol. Nutr. Food Res. 2016; 60: 223-34.

[39] J. M. Goddard, J. H. Hotchkiss. Polymer surface modification for the attachment of bioactive compounds. Progress in Polymer Science 2007; 32:698-725.
[40] L. Bardos, H. Barankova. Cold atmospheric plasma: Sources, processes and applications. Thin Solid Films 2010; 518:670513.

[41] H. Yasuda and M. Gazicki. Biomedical applicatiohs of plasma polymerization and plasma treatment of polymer surfaces. Biomaterials 1982; 3:69-77.

[42] K. S. Chen, T. S. Hung, H. M. Wu, et al. Preparation thermosensitive gold nanoparticles by plasma pretreatment and UV grafted polymerization. Thin Solid Films 2010; 518:7568-37.

[43] Jianping Deng, Lifu Wang, Lianying Liu, et al. Developments and new applications of UV-induced surface draft polymerizations. Progress in Polymer Science 2009; 34:15693.

[44] Dave Mangindaan, Wei-Hsuan Kuo, Ching-Chuan Chang, et al. Plasma polymerization of amine-containing thin films and the studies on the deposition kinetics. Surface \& Coatings Technology 2011; 206:1299-1306.

[45] Annemie Bogaerts, Erik Neyts, Renaat Gijbels, et al. Gas discharge plasma and their applications Spectrochimica Acta Part B 2002; 57:609-18.

[46] M. Morra, E. Occhiello, and F. Garbassi, Contact Angle Hysteresis on Oxgen Plasma Treated Polypropylene Surfaces, Journal of Colloid Interface Science 1989; 132:504-8.

[47] Jay J. Senkevich, Oxidation chemical vapor deposition of polyacenaphthylene, polyacenaphthylene and polyindane via benzoyl peroxide, Thin Solid Films 2014; 556:23-7.

[48] Naohisa Akashi, Shin-ichi Kuroda, Protein immobilization onto poly (vinylidene fluoride) microporous membranes active by the atmospheric pressure low temperature plasma, Polymer 2014; 55:2780-91.

[49] Yashao Chen, Qiang Gao, Haiyan Wan, et al. Surface modification and biocompatible improvement of polystyrene film by $\mathrm{Ar}, \mathrm{O}_{2}$ and $\mathrm{Ar}+\mathrm{O}_{2}$ plasma. Applied Surface Science 2013; 265:452-7.

[50] M. Garcia-Vargas, C. Gonzales-Chomon, B. Magarinos, A. Concheiro, C. Alvarez-Lorenzo, E. Bucio, Acrylic polymergrafted polypropylene sutures for covalent immobilization or reversible adsorption of vancomycin, International Journal of Pharmaceutics 2014; 461:286-95.

[51] Yun Zhao, Alberto Fina, Alberto Venturello, et al. Effect of gas atmospheres on poly (lactic acid) films in acrylic acid plasma treatment. Applied Surface Science 2013; 283:181-7.

[52] Ko-Shao Chen, Yuan-An Ku, Chi-Han Lee, Hong-Ru Lin, Feng-Huei Lin, Tim-Mo Chem, Immobilization of chitosan gel with cross-linking reagent on PNIPAAm gel/PP nonwoven composites surface, Materials Science and Engineering $\mathrm{C}$ $2010 ; 25: 472-8$.

[53] Suseen Jayachandran, Annelies Delabie, Jens Maggen, et al. Chemical vapor deposition processes for the fabrication of epitaxial Si-O superlattices. Thin Solid Films 2014; 557:3641.

[54] Pietro Matricardi, Claudia Cencetti, Roberto Ria, et al. Preparation and Characterization of Novel Gellan Gum Hydrogels Suitable for Modified Drug Release. Molecules 2009; 14:3376-91. 
[55] Yan Xiao, Xiaohua Zhou. Synthesis and properties of a novel crosslinked chitosan resin modified by L-lysine. Reactive \& Functional Polymers 2008; 68:1281-9.

[56] Ganesan Krishnamoorthy, Praveen Kumar Sehgal, Asit Baran Mandal, et al. Novel collagen scaffolds prepared by using unnatural D-amino acid assisted EDC/NHS crosslinking. Journal of Biomaterials Science 2013; 24:344-64.

[57] Chunrong Yang. Enhance physicochemical properties of collagen by using EDC/NHS-crosslinking. Bull. Mater. Sci. 2012; 35:913-8.
[58] J. M. Goddard, J. H. Hotchkiss. Polymer surface modification for the attachment of bioactive compounds. Progress in Polymer Science 2007; 32:698-725.

[59] Vartika Dhyani and Neetu Sigh. Controlling the Cell Adhesion Property of Silk Films by Graft Polymerization. ACS Appl. Mater. Interfaces 2014; 6:5005-11.

[60] William E. Price, Kirsten A. Trickett and Kenneth R. Harris. Association of Caffeine in Aqueous Solution. J. Chem. Soc., Faraday Trans. 1 1989: 85, 3281-8. 